\title{
CDISC SDTM Model Version ResponseTerminology
}

National Cancer Institute

\section{Source}

National Cancer Institute. CDISC SDTM Model Version ResponseTerminology. NCI

Thesaurus. Code C160923.

Terminology associated with the SDT M model version response codelist of the Clinical

Data Interchange Standards Consortium (CDISC) Study Data Tabulation Model (SDT M). 